\title{
Parallel Structure of Feedback Linearization and Sliding Mode Controllers to Track the Desired Velocity Profile in High-Speed Trains
}

\author{
Iman Ferestade ${ }^{(D)},{ }^{1}$ Habibollah Molatefi $\left(\mathbb{D},{ }^{1}\right.$ and Bijan Moaveni ${ }^{2}{ }^{2}$ \\ ${ }^{1}$ Railway Engineering, Iran University of Science and Technology, Tehran, Iran \\ ${ }^{2}$ Electrical Engineering, K. N. Toosi University of Technology, Tehran, Iran \\ Correspondence should be addressed to Habibollah Molatefi; molatefi@iust.ac.ir
}

Received 6 August 2021; Accepted 14 September 2021; Published 18 October 2021

Academic Editor: Xingling Shao

Copyright (C) 2021 Iman Ferestade et al. This is an open access article distributed under the Creative Commons Attribution License, which permits unrestricted use, distribution, and reproduction in any medium, provided the original work is properly cited.

High-speed railway vehicles operate much faster than traditional railway vehicles. After a four-axle high-speed railcar is modeled, an analytical solution is employed in this paper to solve dynamic equations. According to this analytical solution, the coupling of four-axle high-speed railcar equations depends strictly on the adhesion coefficient. A novel parallel control strategy is then formulated to prevent wheels from slipping and track the desired velocity profile. The proposed control strategy includes feedback linearization and sliding mode controllers to achieve the desired performance. Finally, the simulation results indicated the effectiveness of the proposed control system in the high-speed railcar such that the tracking error is less than $12 \%$.

\section{Introduction}

High-speed trains move faster than $200 \mathrm{~km} / \mathrm{h}$ with respect to specific measures [1]. If the slip is exceeded, the acceleration time will increase, something which is undesirable in highspeed trains. Furthermore, trains must work on schedule; thus, every train needs a velocity profile tracker to reach each station at the appropriate time in accordance with the train station schedule.

Yongsong Wei et al. [2] proposed a complete model of longitudinal dynamics for China's high-speed train $(\mathrm{CRH})$ and designed an MPC to track the desired velocity profile up to $80 \mathrm{~m} / \mathrm{s}$. Zhang et al. [3] designed a sliding mode observer and a controller to control the velocity of a high-speed train. They analyzed the effects of the drag coefficient uncertainties and the wind speed. Gao et al. [4] proposed an adaptive neural network control system with a flexible structure for the position and velocity control of high-speed trains. However, the main disadvantage of reviewed methods is that they disregarded the effects of slip between wheels and rails on the wheel's driving force and the locomotive acceleration, although a slip control system is required in the control of the railcar velocity. The slip control system must prohibit more slip in the area of contact between wheels and rails, for this can reduce the tractive force [5] but increase the acceleration time, noise [6], and wheel wear $[7,8]$.

In this paper, a new control system is developed to control the velocity of the four-axle railcar. The control system uses a parallel structure to prevent slip at the beginning of the movement and track the desired velocity profile. To create sufficient tractive force at the beginning of movement, a decentralized slip control system is designed for each axle based on the feedback linearization method for controlling the extent of slip in each axle separately. Moreover, the sliding mode method is employed to track the velocity profile. According to the results, the proposed control system is stable and robust against uncertainties.

This paper consists of four sections. Section 1 presents a literature review and an introduction to the problem. Section 2 presents the dynamic model of a four-axle highspeed railcar by considering some assumptions. In Section 3, an analytical solution is offered to evaluate the effect of the adhesion coefficient on the acceleration of high-speed railcar more accurately. This section is divided into three subsections. Sections 3.1 and 3.2 report the results of the analytical solution in two different cases, whereas Section 3.3 
presents a numerical solution for the validation of analytical results. In Section 4, a control system is designed to control the slip and track the desired velocity profile. Feedback linearization and sliding mode methods are employed in Sections 4.1 and 4.2 to design a slip controller and a velocity profile tracker. The closed-loop results are reported in Section 4.3, whereas validation, stability, and robustness of the proposed controller are evaluated in Sections 4.4, 4.5, and 4.6, respectively.

\section{Modeling the Four-Axle Railcar}

A high-speed multiple unit is a high-speed train on which the drive power is distributed underneath the floors of several railcars. The distribution of power over multiple railcars would allow for higher acceleration than a single locomotive. Different drive power distribution methods can be implemented through high-speed multiple units. Figure 1 demonstrates various types of high-speed multiple units.

According to Figure 1, each railcar is self-propelled in different types of high-speed multiple units. Therefore, the coupling force between the two railcars can be neglected [9]. For the longitudinal dynamic modeling of a four-axle railcar, a free-body diagram of only one railcar can be regarded in Figure 2. The car body is assumed to have no longitudinal movement in proportion to the wheels. Each wheelset is also being rotated independently; hence, the system has five degrees of freedom.

According to Figure 2, motion equations in the longitudinal direction are obtained as follows:

$$
\begin{gathered}
M \ddot{x}_{\mathrm{car}}=F_{a 1}+F_{a 2}+F_{a 3}+F_{a 4}-F_{\text {loss }}, \\
J_{i} \dot{\omega}_{i}=T_{m i}-r_{i} F_{a i}, \quad i=1,2,3,4
\end{gathered}
$$

$$
F_{\text {loss }}=\frac{1}{2} C_{d} A \rho_{\text {air }} V_{\text {car }}^{2}
$$

where $T_{m i}$ refers to the traction motor torque, and $F_{\text {loss }}$ denotes the drag force. According to equations (1) and (2), the dynamic equations of railcars are completely coupled due to the adhesion forces among wheels and rails. Calculated through the following equations, these forces are the major tractive forces in the acceleration and braking systems of a railcar [10]:

$$
\begin{aligned}
F_{a i} & =\mu_{i} \cdot N_{i}, \quad i=1,2,3,4, \\
\mu & =c \cdot e^{\left(-a . v_{s}\right)}-d \cdot e^{\left(-b . v_{s}\right)}, \\
v_{s} & =r . \omega-V_{\mathrm{car}},
\end{aligned}
$$

where $N_{i}$ indicates the normal force, and $\mu_{i}$ shows the adhesion coefficient, whereas $v_{s}$ represents the slip velocity in $\mathrm{km} / \mathrm{h}$. Moreover, parameters $a, b, c$, and $d$ in equation (5) are constant values depending on the conditions of the contact surface between wheels and rails [10]. Figure 3 draws a comparison between equation (5) with experimental results by considering $8 \%$ uncertainty for parameters $a$ and $b$ [11].

According to Figure 3, equation (5) fits the experimental results in the peak area. However, after reaching its maximum value, the curve in equation (5) plunges more quickly than the experimental results curve. Hence, equation (5) presents a good accuracy in the peak area that can be used in the acceleration mode.

The equations of four-axle railcar can be written in the state-space form as follows:

$$
\begin{aligned}
& \dot{z}_{1}=\frac{1}{J_{1}}\left(T_{m 1}-r_{1}\left(\left(c \cdot e^{\left(-3.6 a\left(r_{1} z_{1}-z_{5}\right)\right)}-d \cdot e^{\left(-3.6 b\left(r_{1} z_{1}-z_{5}\right)\right)}\right) N_{1}\right)\right), \\
& \dot{z}_{2}=\frac{1}{J_{2}}\left(T_{m 2}-r_{2}\left(c \cdot e^{\left(-3.6 a\left(r_{2} z_{2}-z_{5}\right)\right)}-d \cdot e^{\left(-3.6 b\left(r_{2} z_{2}-z_{5}\right)\right)} N_{2}\right)\right), \\
& \dot{z}_{3}=\frac{1}{J_{3}}\left(T_{m 3}-r_{3}\left(c \cdot e^{\left(-3.6 a\left(r_{3} z_{3}-z_{5}\right)\right)}-d \cdot e^{\left(-3.6 b\left(r_{3} z_{3}-z_{5}\right)\right)} N_{3}\right)\right), \\
& \dot{z}_{4}=\frac{1}{J_{4}}\left(T_{m 4}-r_{4}\left(c \cdot e^{\left(-3.6 a\left(r_{4} z_{4}-z_{5}\right)\right)}-d \cdot e^{\left(-3.6 b\left(r_{4} z_{4}-z_{5}\right)\right)} N_{4}\right)\right), \\
& \dot{z}_{5}=\frac{1}{M}\left(\sum_{i=1}^{4}\left(\left(c . e^{\left(-3.6 a\left(r_{i} z_{i}-z_{5}\right)\right)}-d . e^{\left(-3.6 b\left(r_{i} z_{i}-z_{5}\right)\right)}\right) N_{i}\right)-\frac{1}{2} C_{d} A \rho_{\mathrm{air}} z_{5}^{2}\right) \\
& z_{1}=\omega_{1}, \\
& z_{2}=\omega_{2}, \\
& z_{3}=\omega_{3}, \\
& z_{4}=\omega_{4}, \\
& z_{5}=V_{\mathrm{car}} .
\end{aligned}
$$




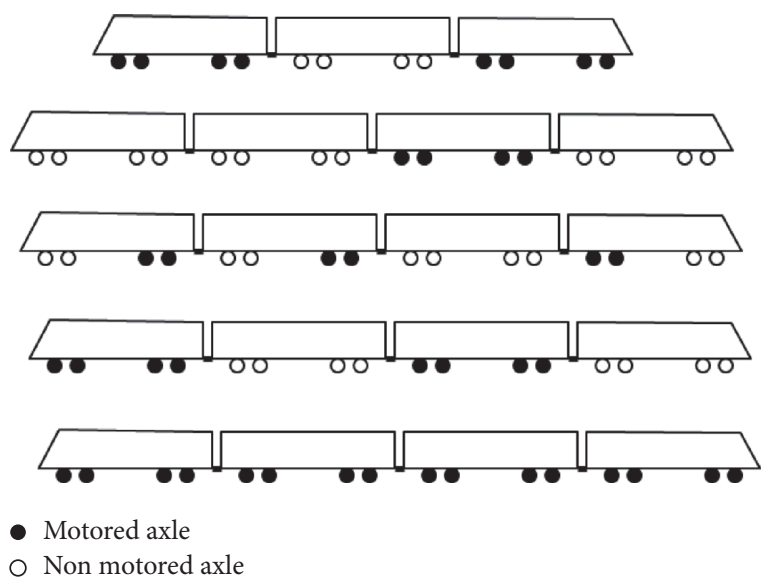

Figure 1: Typical types of high-speed multiple units.

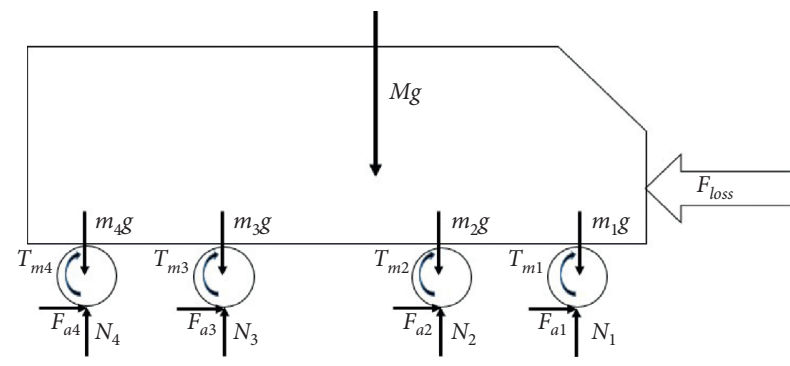

FIGURE 2: The free-body diagram of a four-axle railcar.

\section{Analytical Solution to Dynamic Equations}

The Adomian decomposition method (ADM) is adopted in this section to offer an analytical solution for the state-space model of the system in response to the constant torque. The railcar model is considered a quarter to avoid the calculation complexity of Adomian polynomials for the whole model. By replacing the constant coefficients from Table 1, the statespace model is presented as follows:

$$
\begin{aligned}
\dot{z}_{1}= & -782 e^{-0.89424 z_{1}+1.944 z_{5}}+782 e^{-1.9872 z_{1}+4.32 z_{5}} \\
& +0.01 T_{m}, \\
\dot{z}_{5}= & 10 e^{-0.89424 z_{1}+1.944 z_{5}}-10 e^{-1.9872 z_{1}+4.32 z_{5}} \\
& -0.00009533382353 z_{5}^{2} .
\end{aligned}
$$

The ADM is obtained from equation (8):

$$
\begin{aligned}
& \sum_{n=0}^{\infty} z_{1 n}=\widetilde{z}_{10}+L^{-1}\left(0.01 T_{m}\right)+L^{-1}\left(\sum_{n=0}^{\infty} A_{1 n}\right) \\
& \sum_{n=0}^{\infty} z_{5 n}=\widetilde{z}_{50}+L^{-1}\left(\sum_{n=0}^{\infty} A_{5 n}\right) .
\end{aligned}
$$

After equation (9) is solved through the novel algorithm to determine Adomian polynomials [12], the following equations are obtained:

$$
\begin{aligned}
& z_{1}=z_{10}+z_{11}+z_{12}, \\
& z_{5}=z_{50}+z_{51}+z_{52} .
\end{aligned}
$$

$z_{10}$ to $z_{52}$ are shown in appendix A. According to equation (A.1), the longitudinal velocity of railcar $z_{5}$ and the rotational velocity of wheel $z_{1}$ depend strictly on the traction motor torque. Thus, the results are reported in two cases to reach an in-depth perception of the traction motor torque effect. The first case is the situation in which the traction motor torque is very high (in order of $10^{4}$ ). The other case is a state where the amount of traction motor torque is low (in order of $10^{2}$ ).

3.1. Case I. $\mathbf{T}_{\mathbf{m}}=20000$ N.m. Figure 4 reports the results of Case I.

According to Figure 4, the railcar reaches a very low velocity of $0.0416 \mathrm{~km} / \mathrm{h}$ during $0.008 \mathrm{~s}$ (green curve) due to no appropriate adhesion coefficients. The appropriate adhesion coefficient exists only at the beginning of motion, and its amount reaches zero after nearly $0.008 \mathrm{~s}$. This can also be proven by equation (A.2) which is a simplified version of equation (A.1). According to equation (A.2), coefficients of terms in which $T_{m}$ exists in the equations belonging to $z_{12}$ are in order of $10^{9}$, whereas they are in order of $10^{7}$ for terms of $z_{52}$. Hence, the effect of the input torque on the wheel rotational speed is greater than the railcar velocity. As a result, the adhesion coefficient becomes negligible after $0.008 \mathrm{~s}$.

3.2. Case II. $\mathbf{T}_{\mathbf{m}}=200$ N.m. The ADM solution is not valid when the input torque is 200 N.m due to an increase of adhesion in the contact area between wheels and rails. In other words, once $T_{m}=20000$ (see Figure 4), the adhesion coefficient has a considerable value only at the beginning of motion, whereas it is negligible after nearly $0.008 \mathrm{~s}$. In this case, the coupling of equation (8) decreases, and the presented ADM method is feasible. However, when $T_{m}=200$, the adhesion coefficient has a remarkable value. Consequently, equation (8) is completely coupled.

Hence, it is better to extend the terms carrying power orders in the railcar-state equations until equations exit the power form. The Taylor expansion of order four around the point of $v_{s}=1 \mathrm{~km} / \mathrm{h}$ is employed to extend the power terms of adhesion coefficient relation.

$$
\begin{aligned}
\mu_{\exp }= & \left(e^{-a} c-d e^{-b}\right)+\left(b e^{-b} d-a e^{-a} c\right)\left(v_{s}-1\right) \\
& +\frac{\left(-b^{2} e^{-b} d+a^{2} e^{-a} c\right)\left(v_{s}-1\right)^{2}}{2} \\
& +\frac{\left(b^{3} e^{-b} d-a^{3} e^{-a} c\right)\left(v_{s}-1\right)^{3}}{6}+\frac{\left(-b^{4} e^{-b} d+a^{4} e^{-a} c\right)\left(v_{s}-1\right)^{4}}{24} .
\end{aligned}
$$

Ozis and Yildirım [13] indicated that the ADM method was equivalent to the Homotopy perturbation method (HPM) for equations with exponential nonlinearity in which exponential terms are expanded as the Taylor series [14]. Furthermore, Li [15] illustrated that ADM and HPM were theoretically equivalent in solving nonlinear equations. 

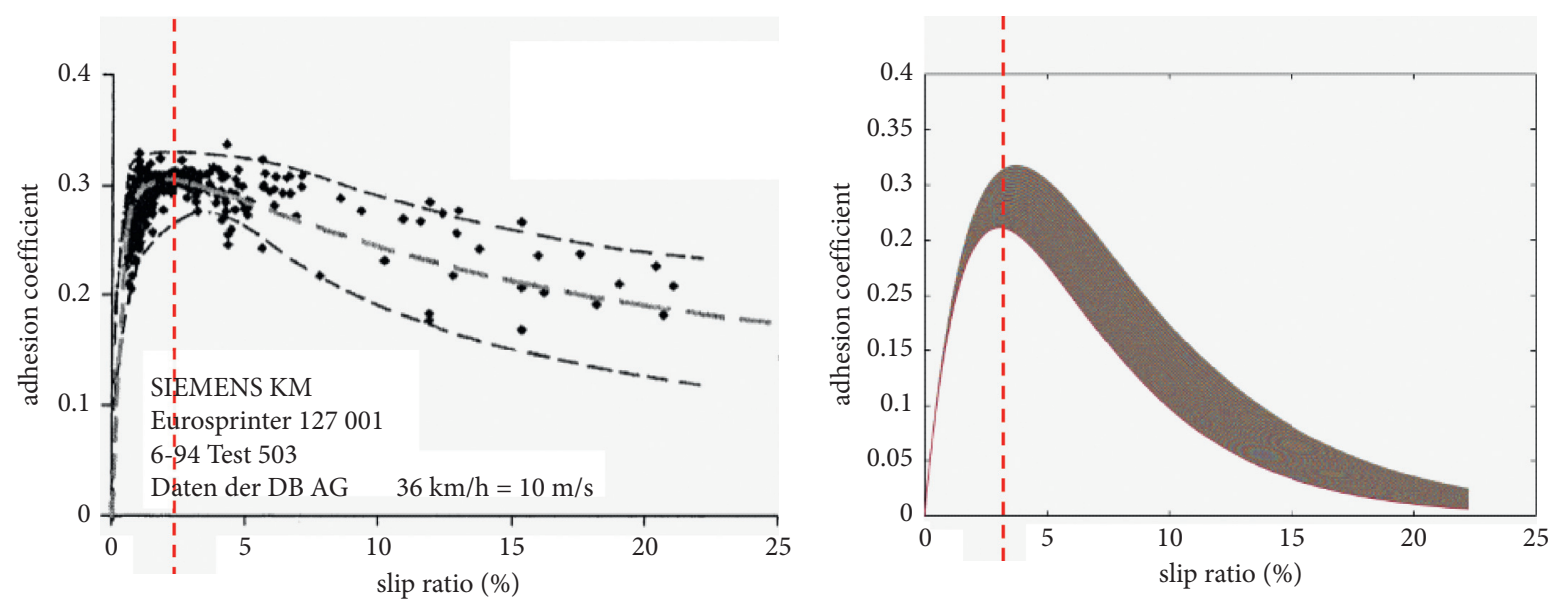

- - - Calculation

- Measurement

(a)

(b)

Figure 3: Comparison between equation (5) and experimental results. (a) Experimental results [11]. (b) Equation (5).

TABLE 1: Characteristics of the ICE3 high-speed railcar.

\begin{tabular}{lcc}
\hline Symbol & Definition (Unit) & Value \\
\hline$M$ & Total mass (tone) & 68 \\
$r$ & Wheel radius (m) & 0.46 \\
$J$ & Axle inertia $\left(\mathrm{kgm}^{2}\right)$ & 100 \\
$C_{d}$ & Coefficient of drag force & 0.25 \\
$A$ & Front surface area of railcar $\left(\mathrm{m}^{2}\right)$ & 10.58 \\
\hline
\end{tabular}
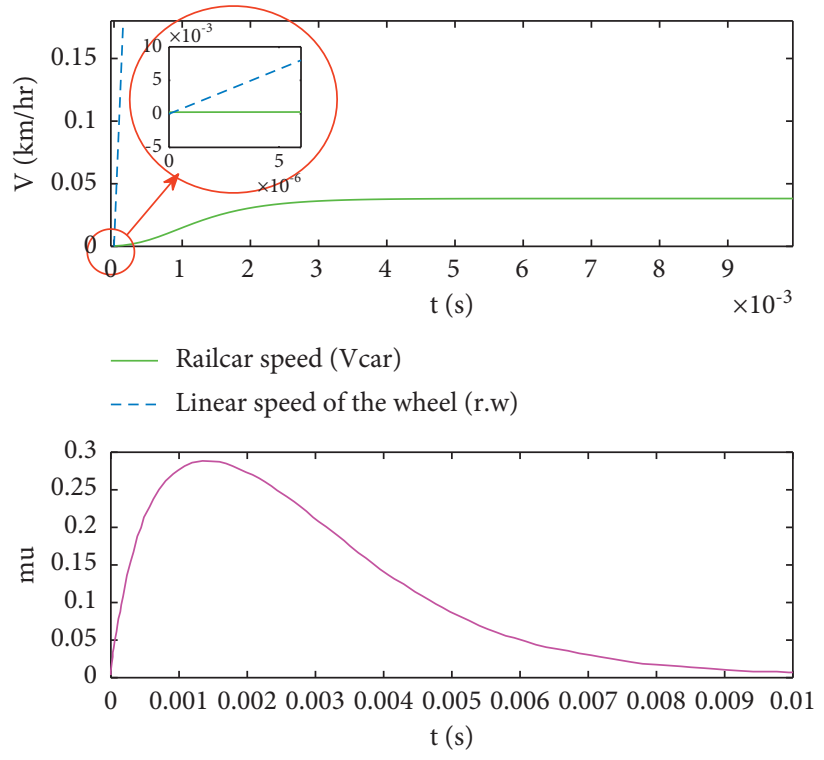

Adhesion coefficient

FIgURE 4: Results of the ADM solution for velocity and adhesion coefficient (Case I). 
Therefore, HPM is employed to solve equation (7) when $T_{m}=200$ N.m.

HPM form of equation (7) can be written as follows:

$$
\begin{aligned}
& (1-p) \frac{\mathrm{d} z_{1}}{\mathrm{~d} t}+p\left(\frac{\mathrm{d} z_{1}}{\mathrm{~d} t}-\frac{1}{J_{w 1}}\left(T_{m 1}-r_{1} \mu_{\exp 1} N_{1}\right)\right)=0, \\
& (1-p) \frac{\mathrm{d} z_{2}}{\mathrm{~d} t}+p\left(\frac{\mathrm{d} z_{2}}{\mathrm{~d} t}-\frac{1}{J_{w 2}}\left(T_{m 2}-r_{2} \mu_{\exp 2} N_{2}\right)\right)=0, \\
& (1-p) \frac{\mathrm{d} z_{3}}{\mathrm{~d} t}+p\left(\frac{\mathrm{d} z_{3}}{\mathrm{~d} t}-\frac{1}{J_{w 3}}\left(T_{m 3}-r_{3} \mu_{\exp 3} N_{3}\right)\right)=0, \\
& (1-p) \frac{\mathrm{d} z_{4}}{\mathrm{~d} t}+p\left(\frac{\mathrm{d} z_{4}}{\mathrm{~d} t}-\frac{1}{J_{w 4}}\left(T_{m 4}-r_{4} \mu_{\exp 4} N_{4}\right)\right)=0, \\
& (1-p) \frac{\mathrm{d} z_{5}}{\mathrm{~d} t}+p\left(\frac{\mathrm{d} z_{5}}{\mathrm{~d} t}-\frac{1}{M}\left(\begin{array}{c}
\mu_{\exp 1} N_{1}+\mu_{\exp 2} N_{2}+\mu_{\exp 3} N_{3} \\
+\mu_{\exp 4} N_{4}-\frac{1}{2} C_{d} A \rho_{\mathrm{air}} z_{5}^{2}
\end{array}\right)\right)=0 .
\end{aligned}
$$

Variables $z_{1}$ to $z_{5}$ of equation (12) are defined as follows:

$$
\begin{aligned}
& z_{1}=z_{10}+p \cdot z_{11}, \\
& z_{2}=z_{20}+p \cdot z_{21}, \\
& z_{3}=z_{30}+p \cdot z_{31}, \\
& z_{4}=z_{40}+p \cdot z_{41}, \\
& z_{5}=z_{50}+p \cdot z_{51} .
\end{aligned}
$$

Due to the oscillations that arise from the existence of microslips, only two sentences in equation (13) are used in order to avoid the complexity of equations. By placing equations (13) into (12), the following results are achieved:

$$
\begin{aligned}
& z_{1}(t)=0.001+0.1724209607 t \\
& z_{2}(t)=0.001+0.1724209607 t \\
& z_{3}(t)=0.001+0.1724209607 t \\
& z_{4}(t)=0.001+0.1724209607 t \\
& z_{5}(t)=0.00036+0.07577712081 t
\end{aligned}
$$

Figure 5 reports the results of the HPM solution for the railcar velocity, the angular velocity of wheels, and the adhesion coefficient for $500 \mathrm{~s}$.

According to Figure 5, the linear speed of the wheel and the railcar velocity are equal. However, since the traction motor input torque is inadequate, the time for acceleration is off the charts. Furthermore, according to equation (14), velocities of railcars and wheels should increase linearly with time; however, it is practically impossible due to the resistance forces. Therefore, it can be concluded that the proposed HPM solution becomes invalid after $500 \mathrm{~s}$. In the HPM solution, it is impossible to calculate the adhesion coefficient from equation (5), and the adhesion coefficient curve in Figure 5 is calculated through a numerical solution.

3.3. Validation of the Analytical Solution. A numerical solution was offered to validate the proposed analytical solutions. Figures 6 and 7 report the results of analytical and numerical solutions in Cases I and II, respectively. Evidently, the numerical and analytical solutions are similar.

Accordingly, the torque results are considerable in a lack of sufficient adhesion for train acceleration. At the same time, a low input torque leads to an increase in the acceleration time and a reduction in the adhesion coefficient. Therefore, a slip control system should be adopted to control the torque input so that the adhesion coefficient is kept in the peak area at the beginning of motion.

\section{Designing the Controlling Structure}

A control system should be designed to control the slip in a way that the maximum adhesion is obtained in the acceleration mode. Whenever a four-axle railcar reaches its proper acceleration, a speed controller is required to track the velocity profile. Thus, the proposed controlling structure consists of two controllers, one of which is utilized to control the amount of adhesion between wheels and rails in the acceleration mode, whereas the other controller is employed for speed control in accordance with the intended velocity profile. For this purpose, a parallel control structure is designed like Figure 8 . In this structure, the feedback linearization method is used for slip control, whereas the sliding mode method is adopted for tracking the desired velocity profile. According to the results, the torque obtained from the slip control system $T_{s i}$ and the torque obtained from the speed control system $T_{v i}$ through $T_{m i}=\alpha T_{s i}+$ $(1-\alpha) T_{v i}, 0<\alpha<1$, were collected together and then exerted on the railcar wheels. The following figure demonstrates the designing trend for each controller.

4.1. Slip Control through Feedback Linearization. By differentiating equation (5) and equating it to zero, the slip velocity allows the highest coefficient of adhesion to be obtained nearly $1.21 \mathrm{~km} / \mathrm{h}$. Therefore, slip control in acceleration aims to reach this slip velocity and consequently reaches the highest coefficient of adhesion. The linearized equations of four-axle railcar movement can be achieved as follows by using the feedback linearization method (see appendix B for details):

$$
\begin{aligned}
& \dot{v}_{s 1}=\chi_{1}, \\
& \dot{v}_{s 2}=\chi_{2}, \\
& \dot{v}_{s 3}=\chi_{3}, \\
& \dot{v}_{s 4}=\chi_{4}, \\
& \dot{\psi}_{1}=\frac{1}{2 M}\left(\begin{array}{c}
-A C_{d} \rho \psi_{1}^{2}+2 N_{1} c e^{-3.6 a v_{s 1}}-2 N_{1} d e^{-3.6 b v_{s 1}} \\
+2 N_{2} c e^{-3.6 a v_{s 2}}-2 N_{2} d e^{-3.6 b v_{s 2}}+2 N_{3} c e^{-3.6 a v_{s 3}} \\
-2 N_{3} d e^{-3.6 b v_{s 3}}+2 N_{4} c e^{-3.6 a v_{s 4}}-2 N_{4} d e^{-3.6 b v_{s 4}}
\end{array}\right),
\end{aligned}
$$

where 


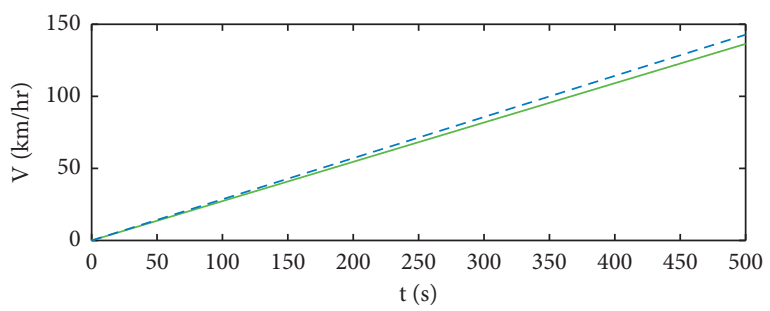

Linea speed of the wheel (r.w)

- - - Railcar speed (Vcar)

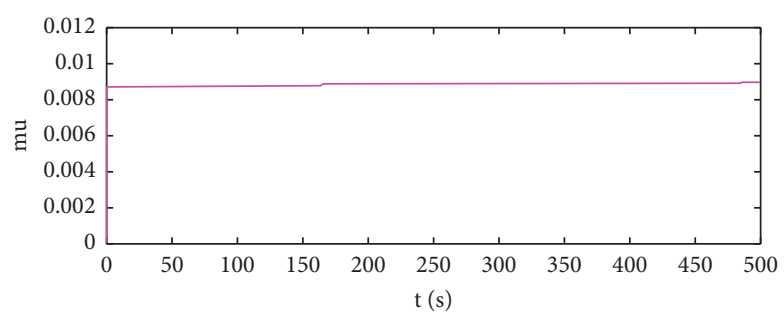

_ Adhesion coefficient

FIgURE 5: Results of the HPM solution for velocity and adhesion coefficient (Case II).

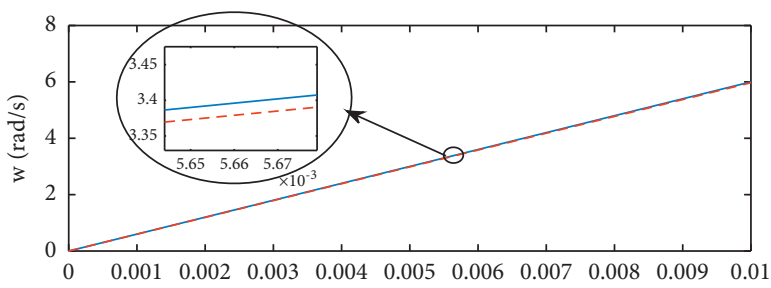

$\mathrm{t}(\mathrm{s})$

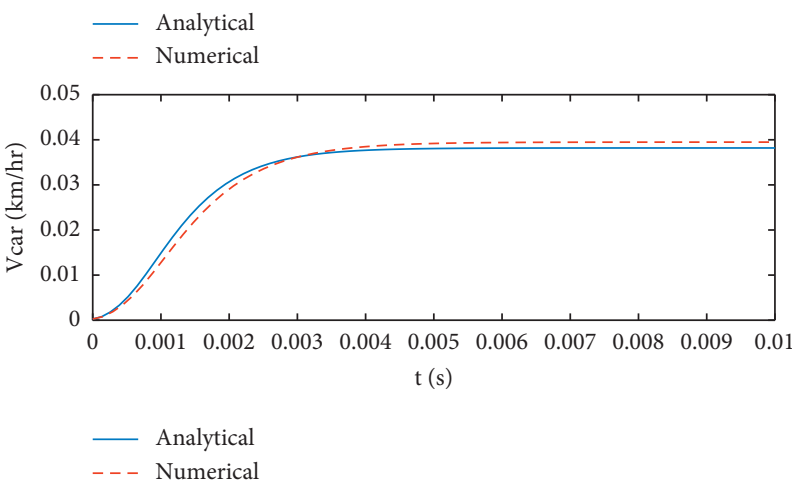

FIgURE 6: Results of numerical and analytical solutions in Case I.

$$
\begin{aligned}
& \chi_{1}=-k_{1} e_{1}, \\
& \chi_{2}=-k_{2} e_{2}, \\
& \chi_{3}=-k_{3} e_{3}, \\
& \chi_{4}=-k_{4} e_{4} .
\end{aligned}
$$

where $e_{1}-e_{4}$ indicate the error between the desired and existent values of slip velocity, and $k_{1}-k_{4}$ depict positive coefficients assuring that this error approaches zero.

4.2. Tracking the Desired Velocity Profile. The safe and efficient operation of a railway system depends mainly on the performance of its automatic train control (ATC) system $[16,17]$. In fact, an ATC system consists of three subsystems, i.e., automatic train operation (ATO), automatic train protection (ATP), and automatic train supervision (ATS) [18], out of which the ATO plays a key role in the urban rail transit system. Generating the desired velocity profile, the ATO tries to track the created velocity profile. Therefore, the ATO can be adopted to help trains perform the operation plan accurately, improve transportation efficiency, enhance the comfort level of passengers, and save energy [19]. Numerous studies have analyzed the ATO design for trains [18-21] and highspeed trains [16]. 

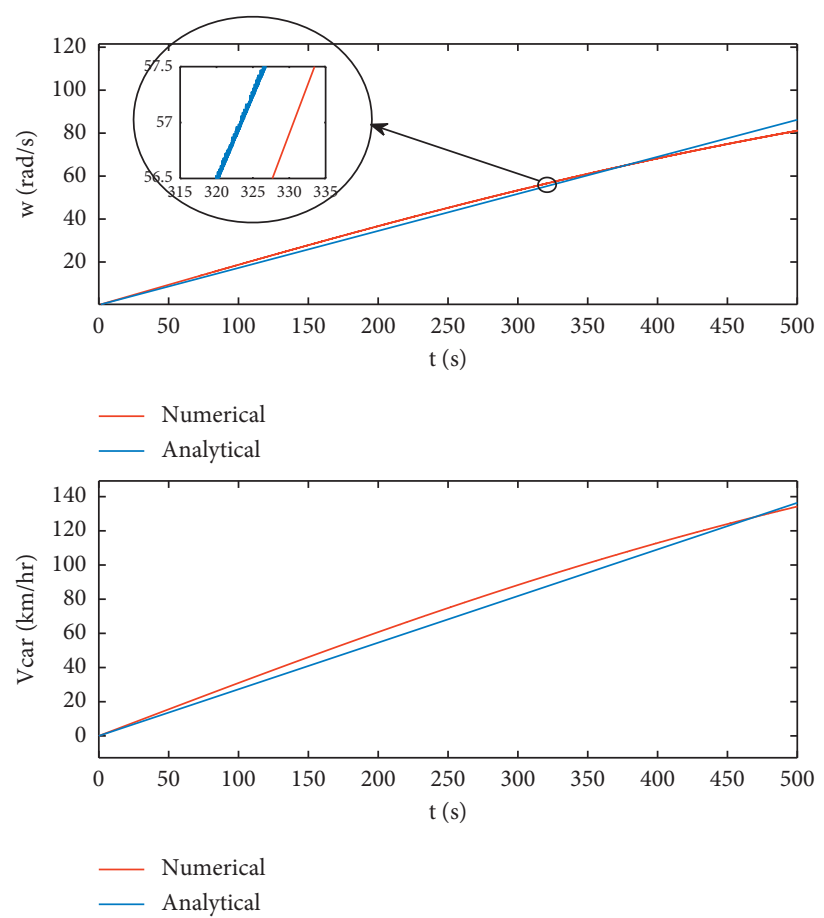

FIgURE 7: Results of numerical and analytical solutions in Case II.

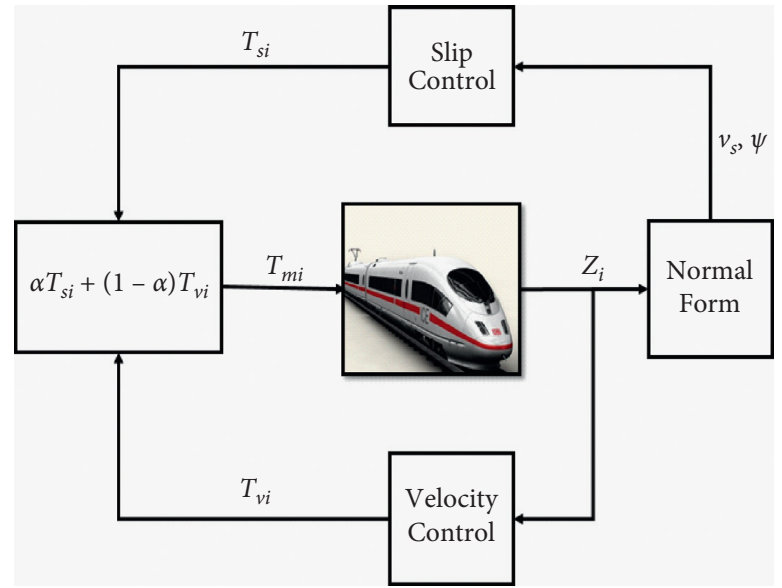

Figure 8: The parallel control structure.

In this section, regarding the velocity profile of a fouraxle ICE3 railcar, a controller is designed to track the desired velocity profile. According to [22], Figure 9 shows the ICE3 profile in acceleration. Evidently, ICE 3 reaches $300 \mathrm{~km} / \mathrm{h}$ in
370 s. To pursue this velocity profile, it should first be estimated by using a function. For this purpose, a seventhorder Gaussian function is used as follows with coefficients shown in Table 2:

$$
\begin{aligned}
V_{d}= & a_{1} \exp \left(-\frac{\left(b_{1}-t\right)^{2}}{c_{1}^{2}}\right)+a_{2} \exp \left(-\frac{\left(b_{2}-t\right)^{2}}{c_{2}^{2}}\right)+a_{3} \exp \left(-\frac{\left(b_{3}-t\right)^{2}}{c_{3}^{2}}\right) \\
& +a_{4} \exp \left(-\frac{\left(b_{4}-t\right)^{2}}{c_{4}^{2}}\right)+a_{5} \exp \left(-\frac{\left(b_{5}-t\right)^{2}}{c_{5}^{2}}\right) \\
& +a_{6} \exp \left(-\frac{\left(b_{6}-t\right)^{2}}{c_{6}^{2}}\right)+a_{7} \exp \left(-\frac{\left(b_{7}-t\right)^{2}}{c_{7}^{2}}\right)
\end{aligned}
$$




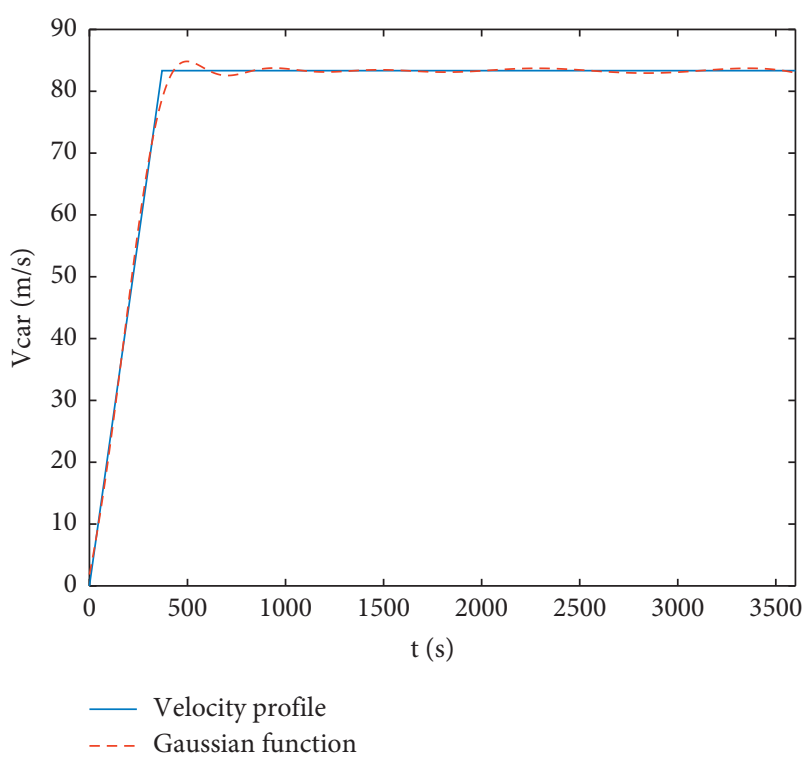

Figure 9: Comparison of the $7^{\text {th }}$ order Gaussian function with the ICE3 velocity profile.

According to Figure 9, this function fits the ICE3 velocity profile properly.

The sliding mode method is employed to track the velocity profile. This method has been implemented successfully in various engineering fields [23-25]. In this study, the
TABle 2: Coefficients of equation (17) for estimation of the ICE3 velocity profile.

\begin{tabular}{lc}
\hline$a_{1}$ & 103.3 \\
$a_{2}$ & -22.2 \\
$a_{5}$ & 56.85 \\
$a_{7}$ & 42.82 \\
$b_{2}$ & $0.3331 e 5$ \\
$b_{4}$ & 471.8 \\
$b_{6}$ & 1837 \\
$c_{1}$ & 1856 \\
$c_{3}$ & 292.5 \\
$c_{5}$ & 443.2 \\
$c_{7}$ & 603.3 \\
$a_{3}$ & 69.36 \\
$a_{4}$ & -33.33 \\
$a_{6}$ & 52.61 \\
$b_{1}$ & 3603 \\
$b_{3}$ & 378 \\
$b_{5}$ & 611.2 \\
$b_{7}$ & 1131 \\
$c_{2}$ & $0.7388 e 7$ \\
$c_{4}$ & 294.9 \\
$c_{6}$ & 965.7 \\
\hline
\end{tabular}

control variable is the railcar velocity, the desired value of which is the velocity profile defined in equation (17). The control input can be obtained from $\dot{s}=\ddot{e}+\lambda \dot{e}=0$ in the following way for the railcar equations:

$$
\begin{aligned}
& T_{v}=u-k \tanh (s),
\end{aligned}
$$

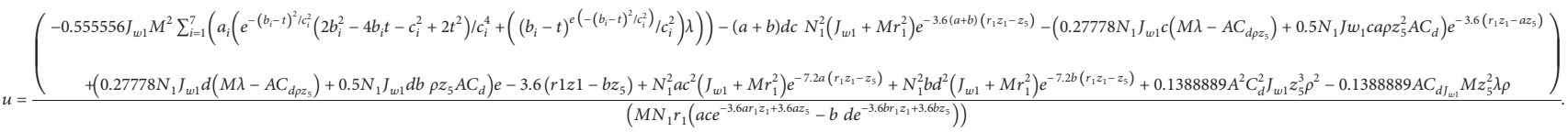

4.3. Simulation and Results. The simulation process was performed within $3600 \mathrm{~s}$. In the first $100 \mathrm{~s}$ requiring appropriate adhesion between wheels and rails for acceleration, $\alpha$ of $T_{m i}=\alpha T_{s i}+(1-\alpha) T_{v i}$ was set at 0.99 . After $100 \mathrm{~s}$ and the creation of proper adhesion between wheels and rails, $\alpha$ was set equal to 0.01 so that the speed control system would track the favorable velocity profile. The limitation for $T_{m i}$ was not to exceed $1980 \mathrm{~N} . \mathrm{m}$, for the traction motor torque of ICE3 was 1980 N.m. Figures 10 and 11 report the results.

According to Figure 10, the ICE3 velocity profile was properly tracked, and the maximum tracking error was reported nearly $12 \%$. Figure 11 shows that the adhesion rate was kept within the 0.0246-0.0251 range due to having a significant portion on the creation of the input torque by the slip control system during the first $500 \mathrm{~s}$. Then, there is a transition zone for the adhesion coefficient so that its value changes from 0.016 to 0.025 due to switching from the slip controller to the velocity profile tracker. Finally, this quantity decreased to nearly 0.0165 by reducing the effect of the slip control system and having no requirement for high adhesion coefficient.

4.4. Validation. An approximate solution can be adopted to verify the results achieved in Subsection 4.3. According to Figure 11, the adhesion coefficient is nearly constant at the beginning and end of motion. Therefore, the following solution can be achieved by using equations (1) to (4) and assuming that the coefficient of adhesion is constant: 


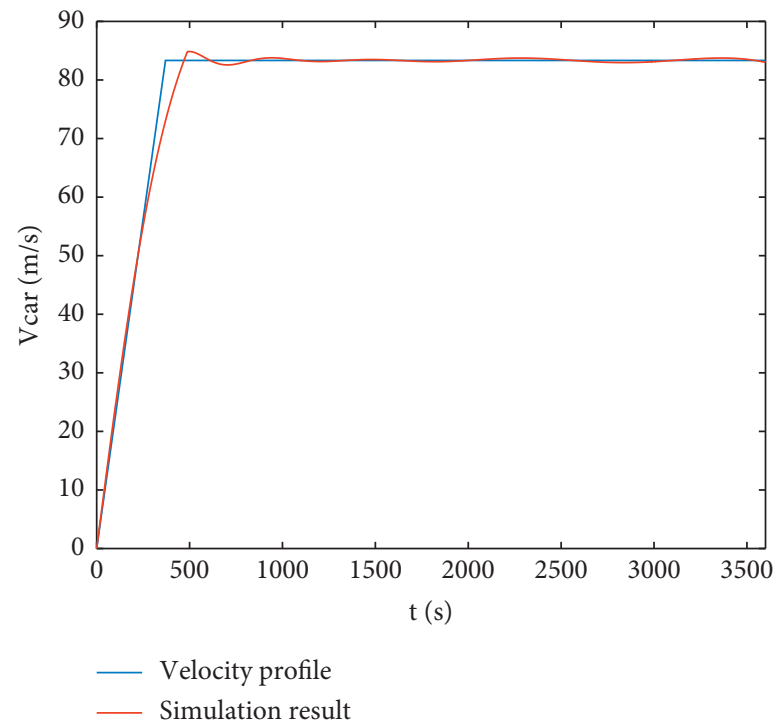

Figure 10: Tracking the velocity profile.

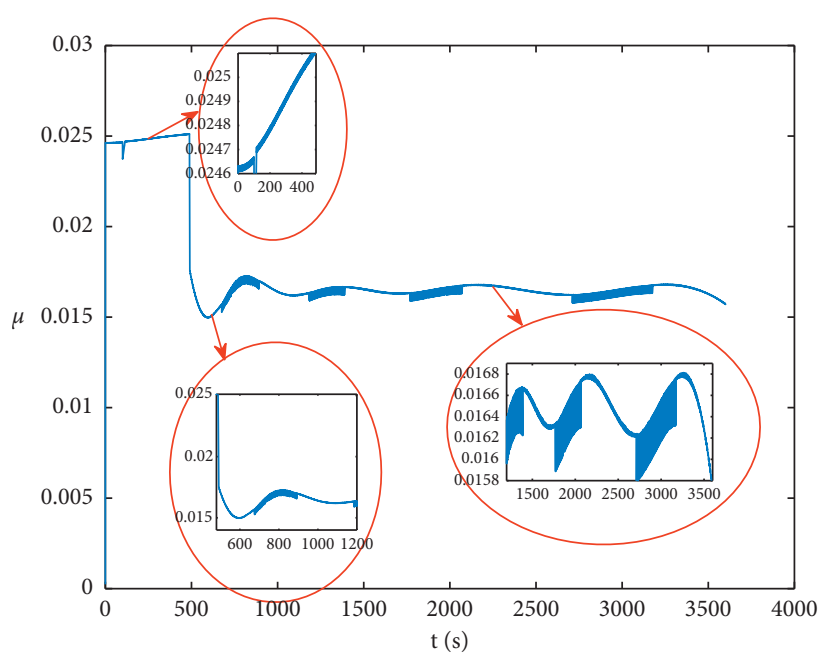

Figure 11: Adhesion coefficient.

$$
V_{\text {car }}=\frac{1}{C_{d} A \rho_{\text {air }}}\left(\tanh \left(\frac{\sqrt{2}}{2 M}\left(\begin{array}{l}
M \sqrt{2} \text { Arctanh } \\
\left(\frac{9 C_{d} A \rho_{\text {air }} \sqrt{2}}{50000 \sqrt{C_{d} A \rho_{\text {air }}\left(\mu_{1} N_{1}+\mu_{2} N_{2}+\mu_{3} N_{3}+\mu_{4} N_{4}\right)}}\right)+ \\
\sqrt{C_{d} A \rho_{\text {air }}\left(\mu_{1} N_{1}+\mu_{2} N_{2}+\mu_{3} N_{3}+\mu_{4} N_{4}\right)} t
\end{array}\right)\right) .\right.
$$

Figure 12 reports the results obtained by placing constant values from Table 1 into equation (19) (the values of $\mu_{1}, \mu_{2}, \mu_{3}, \mu_{4}$ within the range of 0 to $500 \mathrm{~s}$ and 1200 to $3600 \mathrm{~s}$ are considered 0.0249 and 0.0165 , respectively).
In addition, the numerical results are compared with the experimental data of the Chinese high-speed train (CRH3) [26] as well. As it can be seen from Figure 13, the experimental data verify the designed controller performance such 


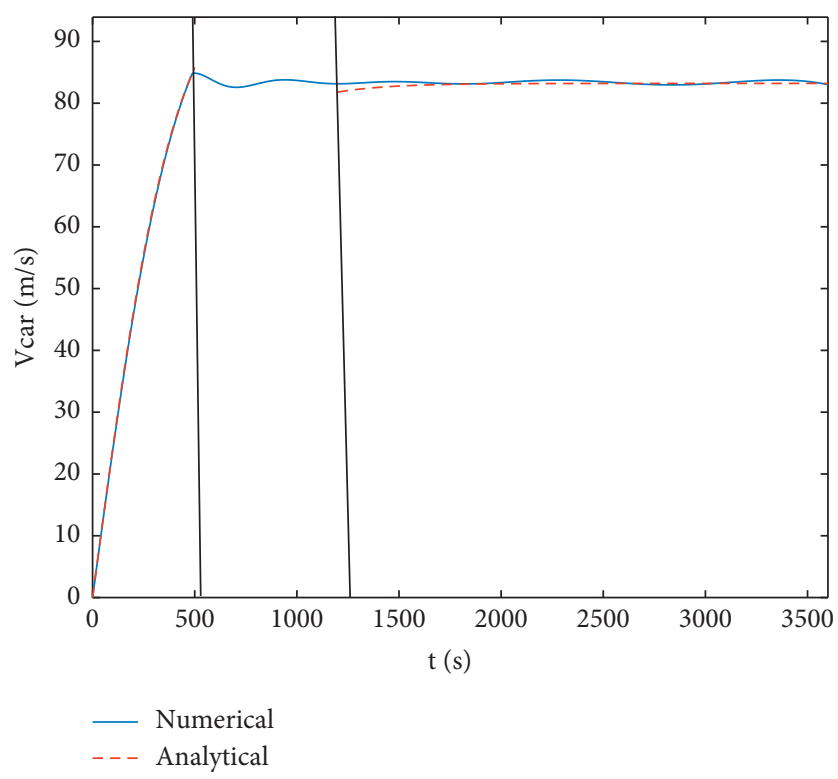

Figure 12: Numerical and analytical solutions for the closed-loop control system.

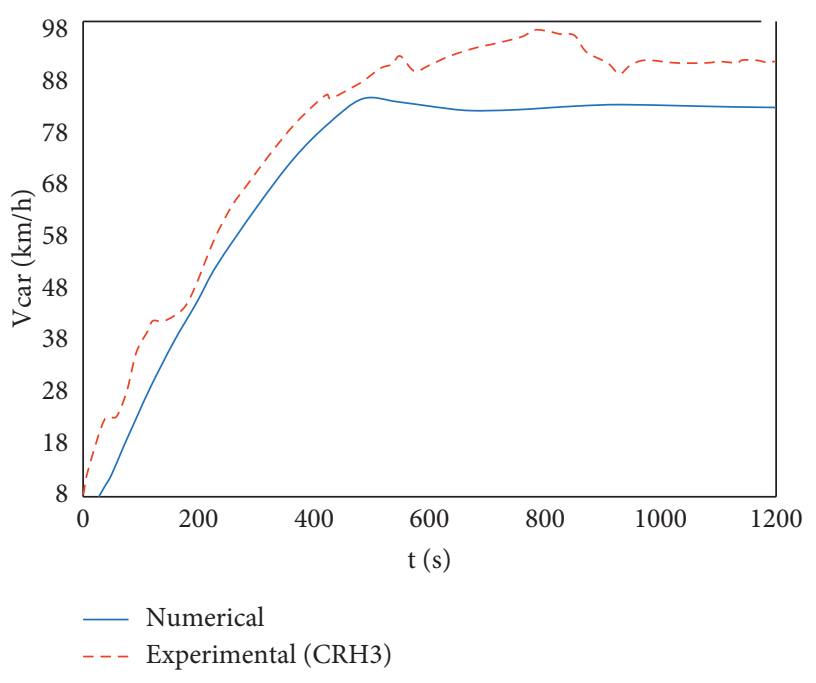

FIgURE 13: Numerical solution and experimental data for the closed-loop control system.

that graphs for numerical methods and experimental data are the same on the transient traction area which is for acceleration. However, the maximum velocity for CRH3 is more which makes the dash-line graph go higher than the solid-line graph in the stable area.

4.5. Stability. The stability of the slip controller should be evaluated to ensure the stability of the proposed control

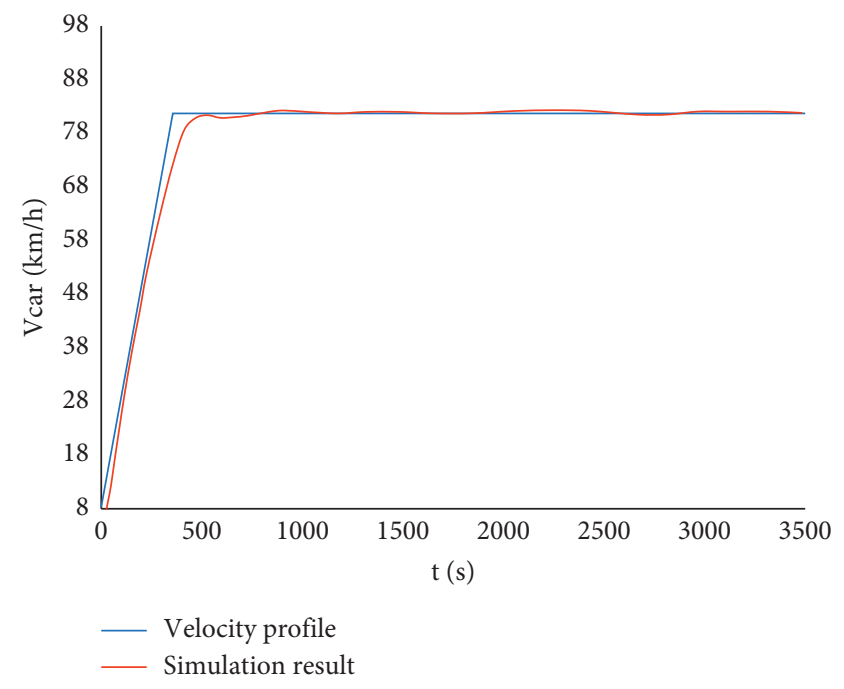

Figure 14: Tracking the velocity profile in Scenario I.

structure. As discussed in Subsection 4.1, the feedback linearization method was employed to design the slip controller. Thus, for the evaluation of stability, it is important to ensure that the internal dynamics of the system are not affected by the input under any circumstances. For this purpose, the zero dynamic stability of the system should first be evaluated. Therefore, in the last equation of equation (B.2) related to $\dot{\psi}_{1}$, all quantities for $v_{s 1}, v_{s 2}, v_{s 3}$, and $v_{s 4}$ are equal to zero to obtain zero dynamics of the system.

$$
\dot{\psi}_{1}=\frac{\left(N_{1}+N_{2}+N_{3}+N_{4}\right) c-\left(N_{1}+N_{2}+N_{3}+N_{4}\right) d-(1 / 2) \rho A C_{d} \psi_{1}^{2}}{M} .
$$




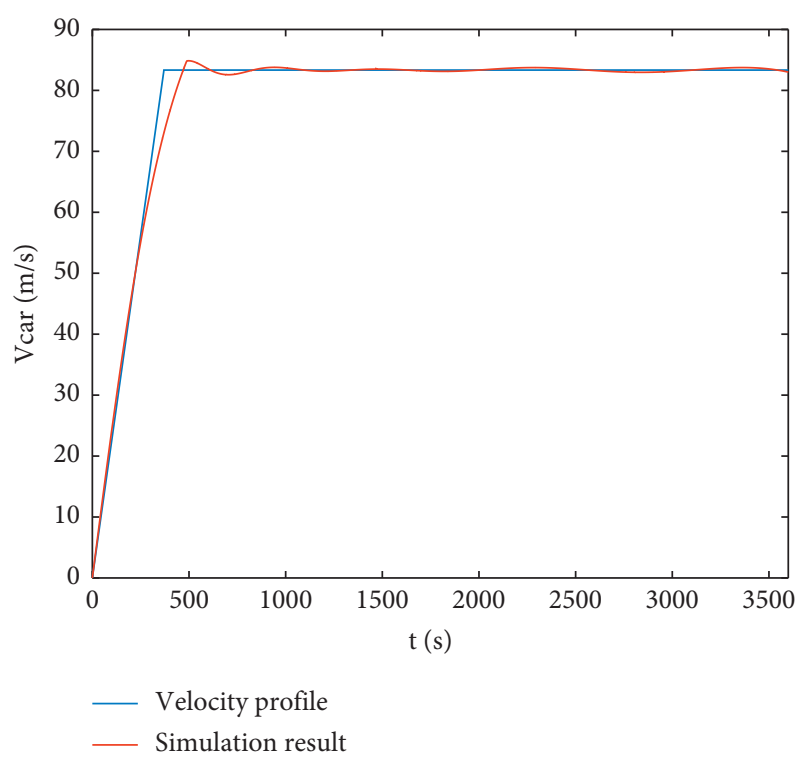

FIgURE 15: Tracking the velocity profile in Scenario II.

The following parameter is obtained by solving equation (20) and using the constant value from Table 1 :

$$
\psi_{1}(t)=\frac{41957.82621}{t} \text {. }
$$

Equation (21) is evidently stable; hence, zero dynamics of the system are stable. Consequently, the internal dynamics of the system are also stable.

4.6. Robustness. In control problem literature, for the evaluation of robustness in the control structure, some unavoidable hardware limits, such as actuator dead zones, are usually studied $[27,28]$. However, for railway vehicle control problems, some specific scenarios for the uncertainty of constant parameters must be considered.

4.6.1. Scenario I. In the first scenario, a wheelset of the fouraxle high-speed railcar is abandoned and replaced with a newer wheelset due to a technical problem. Thus, the new wheelset radius differs from those of the other wheelsets. After the simulation was performed, the following results were achieved by setting the radii of wheelsets at $r_{1}=0.46+0.02, r_{2}=0.46, r_{3}=0.46, r_{4}=0.46$.
According to Figure 14, the control structure can track the desired velocity profile properly even when there are uncertainties in the system parameters.

4.6.2. Scenario II. Although the track was assumed straight with no irregularities, it was possible to consider some uncertainties. Therefore, the second scenario included $N_{1}=$ $170000 \pm 1500, N_{2}=170000 \pm 1500, N_{3}=170000 \pm 1500$, $N_{4}=170000 \pm 1500$.

According to Figure 15, the control structure is robust against uncertainties and can track the desired speed accurately.

\section{Conclusion}

This paper proposed an analytical solution to the motion equations of a four-axle railcar. According to the results, the railcar acceleration time depended on the adhesion coefficient. Therefore, the slip control system is expected to adjust the input torque so that slip remains within the desired range. Consisting of feedback linearization and sliding mode methods, a parallel control structure was designed to control the railcar velocity and track the desired velocity profile. Finally, the results showed that the designed parallel control structure operated properly and that the railcar tracked the velocity profile with less than $12 \%$ tracking error.

In reality, traction motors of high-speed trains are mainly the three-phase asynchronous induction motors, the productive torque of which is a function of flux linkage and current in the rotor and the stator. The flux linkage and current are also functions of the voltage applied to the traction motor by the three-phase power supply network. Therefore, as a future work, a complete model of the dynamic motion of a railcar can be developed to account for the dynamic equations of the traction motor as well. In order to control slip in this complete model, a controller is needed to control the voltage applied to the traction motor.

\section{Appendix}

\section{A. Variables in ADM solution}

$$
\begin{aligned}
& z_{10}=0.001+\frac{1}{100} T_{m} . t \\
& z_{11}=\frac{1}{T_{m}}\left(\begin{array}{c}
-48096.70581+87448.55968 e^{-0.0001944-0.0089424 T_{m} t} \\
-39351.85186 e^{-0.000432-0.019872 T_{m} t}
\end{array}\right),
\end{aligned}
$$




$$
\begin{aligned}
& z_{12}=\frac{1}{T_{m}^{2}}\left(\begin{array}{c}
\left(-0.0000021003948 T_{m} t+3.865720452 \times 10^{9}\right) e^{-0.0001944-0.0089424 T_{m} t} \\
+\left(0.000002100394801 T_{m} t-3.865720451 \times 10^{9}\right) e^{-0.000432-0.019872 T_{m} t} \\
-3.514291466 \times 10^{9} e^{-0.0003888-0.0178848 T_{m} t}+5.828876535 \times 10^{9} \\
e^{-0.0006264-0.0288144 T_{m} t}-1.581431160 \times 10^{9} e^{-0.000864-0.039744 T_{m} t} \\
-7.331539089 \times 10^{8}
\end{array}\right), \\
& z_{50}=0.00036 \text {, } \\
& z_{51}=\frac{1}{T_{m}}\left(\begin{array}{c}
-1.235526353 \times 10^{-11} T_{m} t-1118.268026 e^{-0.0001944-0.0089424 T_{m} t} \\
+503.2206118 e^{-0.000432-0.019872 T_{m} t}+615.0473887
\end{array}\right), \\
& z_{52}=\frac{1}{T_{m}^{2}}\left(\begin{array}{c}
\left(2.685926855 \times 10^{-8} T_{m} t-4.943376536 \times 10^{7}\right) e^{-0.0001944-0.0089424 T_{m} t}+ \\
\left(-2.685926855 \times 10^{-8} T_{m} t+4.943376536 \times 10^{7}\right) e^{-0.000432-0.019872 T_{m} t} \\
-0.00004221706985 T_{m} t+4.493978856 \times 10^{7} e^{-0.0003888-0.0178848 T_{m} t} \\
-7.453806309 \times 10^{7} e^{-0.0006264-0.0288144 T_{m} t}+2.022290486 \\
\times 10^{7} e^{-0.000864-0.039744 T_{m} t}+9.375369668 \times 10^{6}
\end{array}\right)
\end{aligned}
$$

By eliminating smaller terms versus larger ones, equation (A.1) can be simplified as follows:

$$
\begin{aligned}
& z_{10}=0.001+\frac{1}{100} T_{m} \cdot t \\
& z_{11}=\frac{1}{T_{m}}\left(-48096+87448 e^{-0.0089424 T_{m} t}-39351 e^{-0.019872 T_{m} t}\right), \\
& z_{12}=\frac{1}{T_{m}^{2}}\left(\begin{array}{c}
\left.\left(3.8 \times 10^{9}\right) e^{-0.0089424 T_{m} t}+\left(-3.8 \times 10^{9}\right) e^{-0.019872 T_{m} t}-3.5 \times 10^{9} e^{-0.0178848 T_{m} t}\right) \\
z_{50}=0.00036, \quad+10^{9} e^{-0.0288144 T_{m} t}-1.58 \times 10^{9} e^{-0.039744 T_{m} t}-7.3 \times 10^{8}
\end{array}\right), \\
& z_{51}=\frac{1}{T_{m}}\left(-1118.2 e^{-0.0089424 T_{m} t}+503.2 e^{-0.019872 T_{m} t}+615.1\right), \\
& z_{52}=\frac{1}{T_{m}^{2}}\left(\begin{array}{c}
\left(-4.9 \times 10^{7}\right) e^{-0.0089424 T_{m} t}+\left(4.9 \times 10^{7}\right) e^{-0.019872 T_{m} t}+4.4 \times 10^{7} e^{-0.0178848 T_{m} t} \\
-7.4 \times 10^{7} e^{-0.0288144 T_{m} t}+2 \times 10^{7} e^{-0.039744 T_{m} t}+9.3 \times 10^{6}
\end{array}\right) .
\end{aligned}
$$

\section{B. Feedback Linearization Method}

It is possible to formulate state-space equations of the fouraxle railcar in a normal form by adopting the following variables:

$$
\begin{aligned}
& v_{s 1}=r_{1} z_{1}-z_{5}, \\
& v_{s 2}=r_{2} z_{2}-z_{5}, \\
& v_{s 3}=r_{3} z_{3}-z_{5}, \\
& v_{s 4}=r_{4} z_{4}-z_{5}, \\
& \psi_{1}=z_{5} .
\end{aligned}
$$


With the placement of equations (B.1) into (7), the statespace equations for the railcar are created in the normal shape:

$$
\begin{aligned}
& \dot{v}_{s 1}=\frac{1}{2 J_{w 1} M}\left(\begin{array}{c}
-2 c N_{1}\left(M r_{1}^{2}+J_{w 1}\right) e^{-3.6 a v_{s 1}}+2 d N_{1}\left(M r_{1}^{2}+J_{w 1}\right) e^{-3.6 b v_{s 1}} \\
+A C_{d} J_{w 1} \rho \psi_{1}^{2}-2 J_{w 1} N_{2} c e^{-3.6 a v_{s 2}}+2 J_{w 1} N_{2} d e^{-3.6 b v_{s 2}} \\
-2 J_{w 1} N_{3} c e^{-3.6 a v_{s 3}}+2 J_{w 1} N_{3} d e^{-3.6 b v_{s 3}}-2 J_{w 1} N_{4} c e^{-3.6 a v_{s 4}} \\
+2 J_{w 1} N_{4} d e^{-3.6 b v_{s 4}}+2 M r_{1} T_{s 1}
\end{array}\right), \\
& \dot{v}_{s 2}=\frac{1}{2 J_{w 2} M}\left(\begin{array}{c}
-2 c N_{2}\left(M r_{2}^{2}+J_{w 2}\right) e^{-3.6 a v_{s 2}}+2 d N_{2}\left(M r_{2}^{2}+J_{w 2}\right) e^{-3.6 b v_{s 2}} \\
+A C_{d} J_{w 2} \rho \psi_{1}^{2}-2 J_{w 2} N_{3} c e^{-3.6 a v_{s 3}}+2 J_{w 2} N_{3} d e^{-3.6 b v_{s 3}}-2 J_{w 2} N_{4} c e^{-3.6 a v_{s 4}} \\
+2 J_{w 2} N_{4} d e^{-3.6 b v_{s 4}}-2 J_{w 2} N_{1} c e^{-3.6 a v_{s 1}}+2 J_{w 2} N_{1} d e^{-3.6 b v_{s 1}}+2 M r_{2} T_{s 2}
\end{array}\right), \\
& \dot{v}_{s 3}=\frac{1}{2 J_{w 3} M}\left(\begin{array}{c}
-2 c N_{3}\left(M r_{3}^{2}+J_{w 3}\right) e^{-3.6 a v_{s 3}}+2 d N_{3}\left(M r_{3}^{2}+J_{w 3}\right) e^{-3.6 b v_{s 3}} \\
+A C_{d} J_{w 3} \rho \psi_{1}^{2}-2 J_{w 3} N_{2} c e^{-3.6 a v_{s 2}}+2 J_{w 3} N_{2} d e^{-3.6 b v_{s 2}}-2 J_{w 3} N_{4} c e^{-3.6 a v_{s 4}} \\
+2 J_{w 3} N_{4} d e^{-3.6 b v_{s 4}}-2 J_{w 3} N_{1} c e^{-3.6 a v_{s 1}}+2 J_{w 3} N_{1} d e^{-3.6 b v_{s 1}}+2 M r_{3} T_{s 3}
\end{array}\right), \\
& \dot{v}_{s 4}=\frac{1}{2 J_{w 4} M}\left(\begin{array}{c}
-2 c N_{4}\left(M r_{4}^{2}+J_{w 4}\right) e^{-3.6 a v_{s 4}}+2 d N_{4}\left(M r_{4}^{2}+J_{w 4}\right) e^{-3.6 b v_{s 4}} \\
+A C_{d} J_{w 4} \rho \psi_{1}^{2}-2 J_{w 4} N_{3} c e^{-3.6 a v_{s 3}}+2 J_{w 4} N_{3} d e^{-3.6 b v_{s 3}}-2 J_{w 4} N_{2} c e^{-3.6 a v_{s 2}} \\
+2 J_{w 4} N_{2} d e^{-3.6 b v_{s 2}}-2 J_{w 4} N_{1} c e^{-3.6 a v_{s 1}}+2 J_{w 4} N_{1} d e^{-3.6 b v_{s 1}}+2 M r_{4} T_{s 4}
\end{array}\right), \\
& \dot{\psi}_{1}=\frac{1}{2 M}\left(\begin{array}{c}
-A C_{d} \rho \psi_{1}^{2}+2 N_{1} c e^{-3.6 a v_{s 1}}-2 N_{1} d e^{-3.6 b v_{s 1}}+2 N_{2} c e^{-3.6 a v_{s 2}}-2 N_{2} d e^{-3.6 b v_{s 2}} \\
+2 N_{3} c e^{-3.6 a v_{s 3}}-2 N_{3} d e^{-3.6 b v_{s 3}}+2 N_{4} c e^{-3.6 a v_{s 4}}-2 N_{4} d e^{-3.6 b v_{s 4}}
\end{array}\right) \text {, }
\end{aligned}
$$

where $\dot{v}_{s 1}, \dot{v}_{s 2}, \dot{v}_{s 3}$, and $\dot{v}_{s 4}$ are related to the dynamics associated with the linearized part of the system, whereas $\dot{\psi}_{1}$ is associated with internal dynamics.
It is now possible to make the system linear by imposing the input torque as follows:

$$
\begin{aligned}
T_{s 1}=\frac{1}{M r_{1}}\left(\begin{array}{c}
\frac{-1}{2} A C_{d} J_{w 1} \rho \psi_{1}^{2}+c N_{1}\left(M r_{1}^{2}+J_{w 1}\right) e^{-3.6 a v_{s 1}}-d N_{1}\left(M r_{1}^{2}+J_{w 1}\right) \\
e^{-3.6 b v_{s 1}}+J_{w 1} N_{2} c e^{-3.6 a v_{s 2}}-J_{w 1} N_{2} d e^{-3.6 b v_{s 2}}+J_{w 1} N_{3} c e^{-3.6 a v_{s 3}}- \\
J_{w 1} N_{3} d e^{-3.6 b v_{s 3}}+J_{w 1} N_{4} c e^{-3.6 a v_{s 4}}-J_{w 1} N_{4} d e^{-3.6 b v_{s 4}}+J_{w 1} M
\end{array}\right), \\
M r_{2}\left(\begin{array}{c}
\frac{-1}{2} A C_{d} J_{w 1} \rho \psi_{1}^{2}+c N_{2}\left(M r_{2}^{2}+J_{w 2}\right) e^{-3.6 a v_{s 2}}-d N_{2}\left(M r_{2}^{2}+J_{w 2}\right) \\
e^{-3.6 b v_{s 2}}+J_{w 2} N_{3} c e^{-3.6 a v_{s 3}}-J_{w 2} N_{3} d e^{-3.6 b v_{s 3}}+J_{w 2} N_{4} c e^{-3.6 a v_{s 4}}- \\
J_{w 2} N_{4} d e^{-3.6 b v_{s 4}}+J_{w 2} N_{1} c e^{-3.6 a v_{s 1}}-J_{w 2} N_{1} d e^{-3.6 b v_{s 1}}+J_{w 2} M
\end{array}\right),
\end{aligned}
$$




$$
\begin{array}{r}
T_{s 3}=\frac{1}{M r_{3}}\left(\begin{array}{c}
\frac{-1}{2} A C_{d} J_{w 1} \rho \psi_{1}^{2}+c N_{3}\left(M r_{3}^{2}+J_{w 3}\right) e^{-3.6 a v_{s 3}}-d N_{3}\left(M r_{3}^{2}+J_{w 3}\right) \\
e^{-3.6 b v_{s 3}}+J_{w 3} N_{2} c e^{-3.6 a v_{s 2}}-J_{w 3} N_{2} d e^{-3.6 b v_{s 2}}+J_{w 3} N_{4} c e^{-3.6 a v_{s 4}}- \\
J_{w 3} N_{4} d e^{-3.6 b v_{s 4}}+J_{w 3} N_{1} c e^{-3.6 a v_{s 1}}-J_{w 3} N_{1} d e^{-3.6 b v_{s 1}}+J_{w 3} M
\end{array}\right), \\
T_{s 4}=\frac{1}{M r_{4}}\left(\begin{array}{c}
\frac{-1}{2} A C_{d} J_{w 1} \rho \psi_{1}^{2}+c N_{4}\left(M r_{4}^{2}+J_{w 4}\right) e^{-3.6 a v_{s 4}}-d N_{4}\left(M r_{4}^{2}+J_{w 4}\right) \\
e^{-3.6 b v_{s 4}}+J_{w 4} N_{3} c e^{-3.6 a v_{s 3}}-J_{w 4} N_{3} d e^{-3.6 b v_{s 3}}+J_{w 4} N_{2} c e^{-3.6 a v_{s 2}}- \\
J_{w 4} N_{2} d e^{-3.6 b v_{s 2}}+J_{w 4} N_{1} c e^{-3.6 a v_{s 1}}-J_{w 4} N_{1} d e^{-3.6 b v_{s 1}}+J_{w 4} M
\end{array}\right) .
\end{array}
$$

\section{Data Availability}

The output data obtained to support the findings of this study are available from the corresponding author upon request.

\section{Conflicts of Interest}

The authors declare that they have no conflicts of interest.

\section{References}

[1] N. Yanase, Necessities for Future High Speed Rolling Stock, International Union of Railways (UIC), 2010.

[2] Y. Wei, J. Wu, and S. Li, "Modelling and global predictive control for distributed-driven high-speed trains," International Journal of System Control and Information Processing, vol. 1, no. 3, pp. 261-273, 2014.

[3] M. Zhang, Y. Chen, X. Sun, X. Hou, and H. Cai, "Particleoptimized control for automatic train operation based on sliding mode observer," in Proceedings of the 2014 International Conference on Informative and Cybernetics for Computational Social Systems (ICCSS), pp. 79-84, IEEE, Qingdao, China, October 2014.

[4] R.-Z. Gao, Y.-J. Wang, J.-F. Lai, and H. Gao, "Neuro-adaptive fault-tolerant control of high speed trains under tractionbraking failures using self-structuring neural networks," Information Sciences, vol. 367-368, pp. 449-462, 2016.

[5] I. Ferestade, M. Ahmadian, H. Molatefi, B. Moaveni, and V. Bokaeian, "Integrated sliding mode and direct torque controls for improving transient traction in high-speed trains," Journal of Vibration and Control, vol. 27, no. 5-6, pp. 629-650, 2021.

[6] Y. Lyu, E. Bergseth, U. Olofsson, A. Lindgren, and M. Höjer, "On the relationships among wheel-rail surface topography, interface noise and tribological transitions," Wear, vol. 338339, pp. 36-46, 2015.

[7] G. Vasić, F. J. Franklin, and D. I. Fletcher, "Influence of partial slip and direction of traction on wear rate in wheel-rail contact," Wear, vol. 270, pp. 163-171, 2011.

[8] Y. Yang, L. Ling, C. Wang, Z. Liu, K. Wang, and W. Zhai, "Wheel/rail dynamic interaction induced by polygonal wear of locomotive wheels," Vehicle System Dynamics, pp. 1-25, 2020.

[9] W. Shan, L. Wei, and K. Chen, "Longitudinal train dynamics of electric multiple units under rescue," Journal of modern transportation, vol. 25, no. 4, pp. 250-260, 2017.
[10] Y. Ishikawa and A. Kawamura, "Maximum adhesive force control in super high speed train," in Proceedings of the Power Conversion Conference-Nagaoka 1997, pp. 951-954, IEEE, Nagaoka, Japan, August 1997.

[11] B. Engel, H. Beck, and J. Alders, "Verschleißreduzierende radschlupfregelung mit hoher kraftschlussausnutzung," Elektrische Bahnen, vol. 96, pp. 201-209, 1998.

[12] J. Biazar and S. M. Shafiof, "A simple algorithm for calculating adomian polynomials," International Journal of Contemporary Mathematical Sciences, vol. 2, pp. 975-982, 2007.

[13] T. Öziş and A. Yıldırım, "Comparison between Adomian's method and He's homotopy perturbation method," Computers \& Mathematics with Applications, vol. 56, pp. 12161224, 2008.

[14] J.-H. He, "Approximate analytical solution for seepage flow with fractional derivatives in porous media," Computer Methods in Applied Mechanics and Engineering, vol. 167, no. 1-2, pp. 57-68, 1998.

[15] J.-L. Li, "Adomian's decomposition method and homotopy perturbation method in solving nonlinear equations," Journal of Computational and Applied Mathematics, vol. 228, no. 1, pp. 168-173, 2009.

[16] H. Dong, B. Ning, B. Cai, and Z. Hou, "Automatic train control system development and simulation for high-speed railways," IEEE Circuits and Systems Magazine, vol. 10, no. 2, pp. 6-18, 2010.

[17] B. Suthar and A. Patel, "Design optimization of axial flux surface mounted permanent magnet brushless dc motor for electrical vehicle based on genetic algorithm," International Journal of Engineering, vol. 31, pp. 1050-1056, 2018.

[18] H. Sun, Z. Hou, and T. Tang, "An iterative learning approach for train trajectory tracking Control," in IFAC Proceedings Volumesvol. 44, no. 1, , pp. 14916-14921, International Federation of Automatic Control, 2011.

[19] X. Zhu, R. Zhang, W. Dai, Z. Zhang, and J. Li, "Performance and safety assessment of ATO systems in urban rail transit systems in China," Journal of Transportation Engineering, vol. 139, no. 7, pp. 728-737, 2013.

[20] J. Yang, L. Jia, S. Lu, Y. Fu, and J. Ge, "Energy-efficient speed profile Approximation: an optimal switching region-based approach with adaptive resolution," Energies, vol. 9, no. 10, p. 762, 2016.

[21] L. Fan, F. Cao, B. Ke, and T. Tang, "Velocity profile optimization for train operation based on ant colony algorithm," in Proceedings of the Advanced Information Technology, Electronic and Automation Control Conference (IAEAC), pp. 48-53, IEEE, Chongqing, China, December 2015. 
[22] R. Liu and Y. Deng, Engineering Comparison of High-Speed Rail and Maglev Systems: A Case Study of Beijing-Shanghai Corridor, The Transportation Research Board, Washington, DC, USA, 2004.

[23] W. Wang, D. Mao, and B. Li, "Extend disturbance observer of backlash-based robust sliding-mode control," Mathematical Problems in Engineering, vol. 2021, Article ID 8782813, 17 pages, 2021.

[24] R. Yang, Y. Fu, L. Zhang, H. Qi, X. Han, and J. Fu, "A novel sliding mode control framework for electrohydrostatic position actuation system," Mathematical Problems in Engineering, vol. 2018, Article ID 7159891, 22 pages, 2018.

[25] R. Wang, L. Gao, Ch. Bai, and H. Sun, "U-Model-Based sliding mode controller design for quadrotor UAV control systems," Mathematical Problems in Engineering, vol. 2020, Article ID 4343214, 11 pages, 2020.

[26] P. Connor, "High speed railway capacity: understanding the factors affecting capacity limits for a high speed railway," in Proceedings of the International Conference on High Speed Rail: Celebrating Ambition, University of Birmingham, Birmingham, England, 2014.

[27] T. Yang, N. Sun, and Y. Fang, "Adaptive fuzzy control for uncertain mechatronic systems with state estimation and input nonlinearities," IEEE Transactions on Industrial Informatics 1 page, 2021

[28] T. Yang, N. Sun, and Y. Fang, "Adaptive fuzzy control for a class of MIMO underactuated systems with plant uncertainties and actuator deadzones: design and experiments," IEEE Transactions on Cybernetics, pp. 1-14, 2021. 